\title{
COMPLICAÇÕES NOS PRIMEIROS 30 DIAS PÓS-TRANSPLANTE HEPÁTICO - INSTRUMENTO PARA AVALIAÇÃO NO ÂMBITO DO SISTEMA ESTADUAL DE TRANSPLANTES DO PARANÁ
}

\author{
Liver transplant complications in the first 30 days - assessment tool of the transplant \\ system in the state of Parana
}

Cassia Regina Sbrissia Silveira, Fábio Silveira, Fábio Porto Silveira, Nestor Saucedo Júnior

\section{RESUMO}

Objetivo: Identificar e graduar as complicações ocorridas durante os 30 primeiros dias dos transplantes hepáticos com doador falecido (THF), utilizando a classificação de Clavien-Dindo, segundo os escores DRI, P-SOFT, SOFT, MELD e BAR. Propor adoção de acompanhamento prospectivo desses dados no âmbito de todo o Sistema Estadual de Transplantes do Paraná. Métodos: Coorte observacional dos casos de THF do programa do Hospital do Rocio, Campo Largo, Paraná, nos anos de 2016 e 2017. Resultados: Significativo aumento do índice de gravidade representado pelo MELD, com 20,2 $\pm 4,8$ (2016) e 24,4 $\pm 7,11(2017)$, com $p=0,0076$. Inexistência de diferenças significativas do BAR $(8,03 \pm 3,86$ vs $9,54 \pm 4,11)$, PSOFT $(12,38 \pm 6,43$ vs $11,83 \pm 7,83)$ e SOFT $(12,32 \pm 7,6)$. Aumento da incidência de complicações Grau IVb e V. Frequência de mortalidade cirúrgica de 9,68\%, em 2016, e de 35,85\%, em 2017. Conclusão: Houve aumento da gravidade de receptores representados pelo MELD, com aumento de mortalidade em razão de complicações infecciosas. A adoção de acompanhamento prospectivo dos dados de transplante hepático no estado do Paraná, com projeções dos subgrupos de gravidade, segundo os índices adotados, servirão de base crítica para melhora contínua, transparência e melhor direcionamento de recursos do Sistema de Saúde.

Descritores: Transplante de Fígado; Índice de Gravidade de Doença; Taxa de Sobrevida; Benchmarking

Instituição:

Departamento de Transplante Hepático do Hospital do Rocio, Campo Largo/PR - Brasil.

\section{Correspondência:}

Fábio Silveira

End.: Rua Voluntários da Pátria, 475, S/905 A, Curitiba/PR - Brasil Tel.: (41) 3223-2219

E-mail: silveira.fabio@gmail.com

Recebido em: 25/06/2018

Aceito em: 13/07/2018

\section{INTRODUÇÃO}

Já bem estabelecido como o tratamento mais adequado para as doenças hepáticas terminais, o transplante hepático envolve uma miríade de fatores relacionados ao doador, receptor, ato cirúrgico, anestesiológico e de manejo em terapia intensiva que influenciam a ocorrência das complicações cirúrgicas. ${ }^{1}$

As Diretrizes da Política Estadual de Transplantes do Paraná foram formuladas com o objetivo de reorganizar e otimizar o Sistema Estadual de Transplantes do estado, tendo como principal objetivo o aumento do número de notificações, doações e o número de transplantes. Com os últimos atingidos, a sequência estratégica envolve o estabelecimento de parâmetros e indicadores dos 
resultados desejados para monitoramento e avaliação dos serviços. ${ }^{2}$

Definida como um desvio de um curso pós-operatório normal não inerente ao procedimento, a complicação cirúrgica resulta em aumento no risco de morbidade e mortalidade, após o transplante hepático, com aumento de custos e do risco da perda do enxerto. ${ }^{3}$

O nebuloso território da falta de dados para uma análise correta dos desfechos do transplante não permite o conhecimento da realidade estadual, com suas multifacetadas virtudes e dificuldades, deixando margem a interpretações não embasadas e, por muitas vezes, deletérias ao sistema.

A adoção de critérios objetivos para a classificação das complicações pós-operatórias, como a classificação de Clavien-Dindo ${ }^{4} \mathrm{e}$ de escores validados como o DRI, ${ }^{5}$ P-SOFT / SOFT, ${ }^{6}$ MELD ${ }^{7}$ e BAR ${ }^{8}$, para a avaliação dos conhecidos fatores de risco do transplante hepático, pode auxiliar-nos na dissipação dessa nebulosidade.

O presente estudo tem por objetivo (A) identificar e graduar as complicações ocorridas durante os 30 primeiros dias dos transplantes hepáticos com doador falecido (THF), utilizando a classificação de ClavienDindo, segundo os escores DRI, P-SOFT, SOFT, MELD e BAR; e (B) propor adoção de acompanhamento prospectivo desses dados no âmbito de todo o Sistema Estadual de Transplantes do Paraná.

\section{MÉTODOS}

Coorte observacional dos casos de THF do programa do Hospital do Rocio, Campo Largo, Paraná. O período da análise compreende de 01/11/2015 a 30/11/2017. O primeiro período, chamado 2016, compreende a data do primeiro caso até 30 de novembro de 2016. O segundo período, 2017, até 30 de novembro de 2017. Houve 82 casos de transplante de fígado isolado, sendo dois casos de transplante duplo de fígado-rim.

Tendo em vista que a maioria das complicações após THF ocorrem de maneira precoce ${ }^{9}$ no pós-operatório, juntamente com a probabilidade de falta de acurácia das informações prestadas após os primeiros meses, em uma esfera estadual, optamos por examinar as complicações ocorridas durante os 30 primeiros dias do procedimento.

Em casos de pacientes que necessitaram de retransplante nos primeiros 30 dias, as complicações que ocorreram até o retransplante, incluindo a perda do enxerto, foram incluídas, mas todas as outras complicações subsequentes, exceto pela sobrevida do paciente, foram consideradas censuradas.
Como desfecho primário, foi considerado o número de complicações por paciente e a ocorrência de complicações graves ( $\geq$ Grau III). Desfecho secundário foi considerado o óbito dentro dos primeiros 30 dias do transplante (complicação grau $\mathrm{V}$ ), sobrevida do enxerto e do paciente.

Como fatores de risco do receptor, foram considerados a idade, sexo, diagnóstico e índice de massa corpórea, cálculos do MELD e MELD ajustado para situações especiais, ambos com os valores válidos no Sistema Nacional de Transplante (SNT) da data de alocação do órgão para o paciente.

Como fatores de risco do doador, que compõem o DRI, foram considerados a idade, altura $(\mathrm{cm})$, causa do óbito, raça, local da doação, número de dias de internamento em UTI e valor da creatinina sérica da data da oferta do órgão.

Os fatores de risco do binômio doador-receptor que compõem os escores PSOFT, SOFT e BAR foram os seguintes: número de transplante prévios, cirurgia prévia em andar superior de abdome (excluindo colecistectomia e fundoplicatura vídeo-laparoscópica), albumina sérica, necessidade de diálise no pré-transplante, internamento em UTI ou enfermaria no momento do transplante, trombose de veia porta, presença de ascite e encefalopatia, suporte de vida com ventilação mecânica no momento do transplante, sangramento venoso portal nas $48 \mathrm{~h}$ anteriores ao procedimento e tempo de isquemia fria.

O sistema de classificação de Clavien-Dindo ${ }^{4}$ foi utilizado para identificação e estratificação das complicações. Da mesma maneira, foram considerados normais, como parte da recuperação pós-operatória e doravante não considerados: necessidade de ventilação mecânica até $48 \mathrm{~h}$ após o término da cirurgia, fluidos IV para hipotensão transitória, utilização de medicamentos para náuseas e vômitos, suporte enteral / parenteral até sete dias após a cirurgia, transfusões de até $4 U$ de concentrado de hemácias nas primeiras $48 \mathrm{~h}$, assim como plaquetas ou plasma fresco congelado. Ajuste rotineiro das doses das medicações imunossupressoras não são complicações. Mudança de imunossupressor secundária a efeitos colaterais do primeiro foi considerada complicação. Testes não invasivos como ultrassonografia/doppler, tomografia, ressonância não foram considerados complicações. Modificação do uso de antibiótico, além de profilaxia foi considerada complicação grau II. Biópsia hepática foi considerada complicação IIIA, independente do resultado da biópsia.

A coleta de dados deu-se de maneira prospectiva, com a utilização de software de base de dados e estatística Epi-Info (Center for Disease Control-USA). ${ }^{10}$ Para os cálculos de significância estatística, utilizou-se o teste paramétrico de ANOVA, adotando-se o valor de $p<0,05$. 


\section{RESULTADOS}

Um total de 84 transplantes foram avaliados, sendo 31 em 2016 e 53 em 2017. As características demográficas dos pacientes estão demonstradas na tabela 1.

Tabela 1 - Características demográficas.

\begin{tabular}{lcc}
\hline & 2016 & 2017 \\
\hline Idade (anos) & $49,2 \pm 10,7$ & $50,4 \pm 11,7$ \\
\% feminino & $41,94 \%$ & $35,85 \%$ \\
\% brancos & $61,29 \%$ & $69,81 \%$ \\
Altura (m) & $1.68 \pm 0.08$ & $1.67 \pm 0.08$ \\
Peso (kg) & $75.7 \pm 17.9$ & $74.9 \pm 13.5$ \\
Índice de massa corpórea & $26.64 \pm 5.78$ & $26.65 \pm 4$ \\
Etiologia & & \\
$\quad$ Álcool & $16,13 \%$ & $23,8 \%$ \\
Criptogênica & $19,35 \%$ & $19,23 \%$ \\
Vírus C & $16,13 \%$ & $7,69 \%$ \\
EHNA & - & $3,85 \%$ \\
Doença colestática & $16,13 \%$ & $15,38 \%$ \\
Vírus B & $12,9 \%$ & $13,46 \%$ \\
Insuficiência hepática aguda & $6,45 \%$ & $3,85 \%$ \\
Malignidade & $6,45 \%$ & $7,69 \%$ \\
Não funcionamento primário & $6,45 \%$ & - \\
Tempo espera lista (dias) & $73,8 \pm 89,2$ & $86,4 \pm 118,05$ \\
\hline
\end{tabular}

Análise da gravidade dos receptores representado pelo MELD (Tabela 2) e ambiente de internamento prétransplante (Figura 1).

Tabela 2 - Análise de gravidade - receptores

\begin{tabular}{lccc}
\hline & $\mathbf{2 0 1 6}$ & $\mathbf{2 0 1 7}$ & $\mathbf{p}$ \\
\hline MELD & $20,2 \pm 4,8$ & $24,4 \pm 7,11$ & $0,0076^{*}$ \\
CHILD C (\%) & $64,52 \%$ & $63,46 \%$ & - \\
Exceção MELD & $6,46 \%$ & $7,69 \%$ & - \\
\hline
\end{tabular}

Figura 1 - Status clínico do receptor no momento do transplante. (\%)

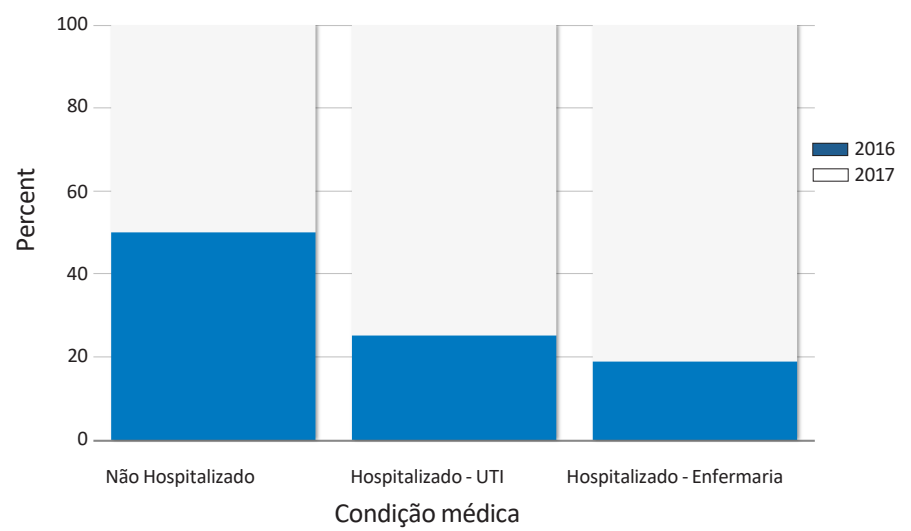

Características do doador, analisadas pelo índice de risco do doador (donor risk index - DRI), avaliação sequencial de falência orgânica (sequential organ failure assessment - SOFA) e o tempo de isquemia fria estão demonstrados na tabela 3 .

Tabela 3 - Análise de gravidade - doadores

\begin{tabular}{lccc}
\hline & $\mathbf{2 0 1 6}$ & $\mathbf{2 0 1 7}$ & $\mathbf{p}$ \\
\hline Idade & $35,67 \pm 15,48$ & $39,90 \pm 15,91$ & 0,29 \\
DRI & $1,37 \pm 0,32$ & $1,39 \pm 0,31$ & 0,82 \\
SOFA & $10,29 \pm 2$ & $10,01 \pm 2,37$ & 0,74 \\
Isquemia fria (minutos) & $437,93 \pm 104$ & $449,59 \pm 98,98$ & 0,70 \\
\hline
\end{tabular}

Índices de gravidade envolvendo fatores relacionados ao receptor e doador, BAR, PSOFT, SOFT e DMELD estão demonstrados na tabela 4.

Tabela 4 - Análise de gravidade, binômio doador - receptor

\begin{tabular}{lccc}
\hline & $\mathbf{2 0 1 6}$ & $\mathbf{2 0 1 7}$ & $\mathbf{p}$ \\
\hline BAR & $8,03 \pm 3,86$ & $9,54 \pm 4,11$ & 0,71 \\
DMELD & $765 \pm 395,7$ & $954,1 \pm 400,5$ & $0,0473^{*}$ \\
PSOFT & $12,38 \pm 6,43$ & $11,83 \pm 7,83$ & 0,49 \\
SOFT & $12,32 \pm 7,6$ & $12,79 \pm 8,23$ & 0,94 \\
\hline
\end{tabular}

Complicações estratificadas segundo Clavien-Dindo, ocorreram conforme a figura 2 .

Figura 2 - Complicações cirúrgicas segundo Clavien-Dindo

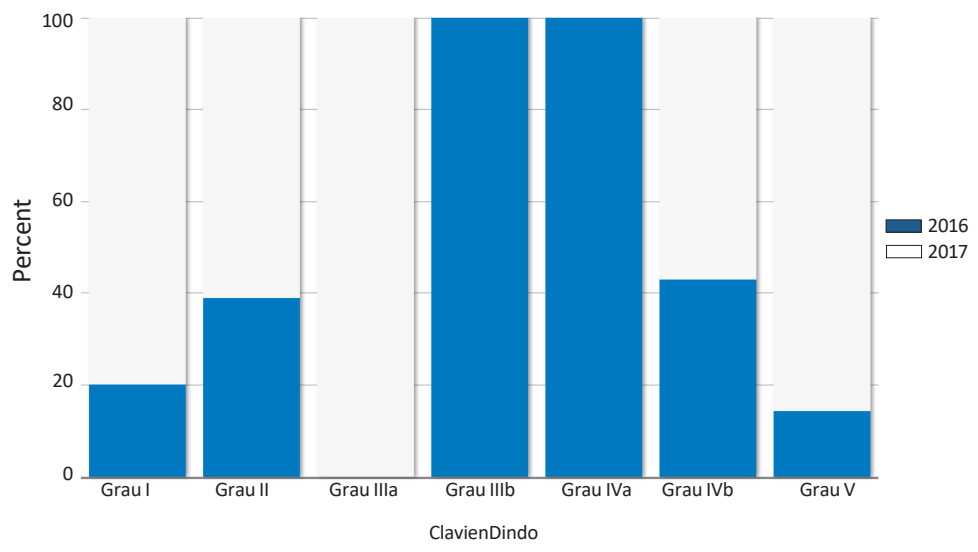

A incidência de complicações cirúrgicas, específicas do transplante de fígado, está demonstrada na tabela 5. 
A frequência de mortalidade cirúrgica foi de $9,68 \%$ no período de 2016 , e de $35,85 \%$ no período de 2017 . Dos pacientes que não apresentaram mortalidade cirúrgica, a sobrevida manteve-se em taxas de $78 \%$ em ambos os períodos, com seguimento médio de 206,23 dias.

A aplicação do diagrama de Pareto aos motivos de mortalidade cirúrgica está demonstrada na figura 3.

Tabela 5 - Complicações cirúrgicas

\begin{tabular}{lcc}
\hline & $\mathbf{2 0 1 6}$ & $\mathbf{2 0 1 7}$ \\
\hline Reoperações & $20 \%(n=6)$ & $13,04 \%(n=6)$ \\
Trombose arterial & - & $5,6 \%(n=3)$ \\
Complicações biliares & $22,58 \%(n=7)$ & $7,55 \%(n=4)$ \\
Re-transplante & $6,45 \%(n=2)$ & $1,92 \%(n=1)$ \\
Disfunção inicial do enxerto & $12,9 \%(n=4)$ & $7,55 \%(n=4)$ \\
Não-funcionamento primário do enxerto & $9,68 \%(n=3)$ & - \\
\hline
\end{tabular}

Figura 3 - Diagrama de Pareto para as causas de mortalidade cirúrgica.

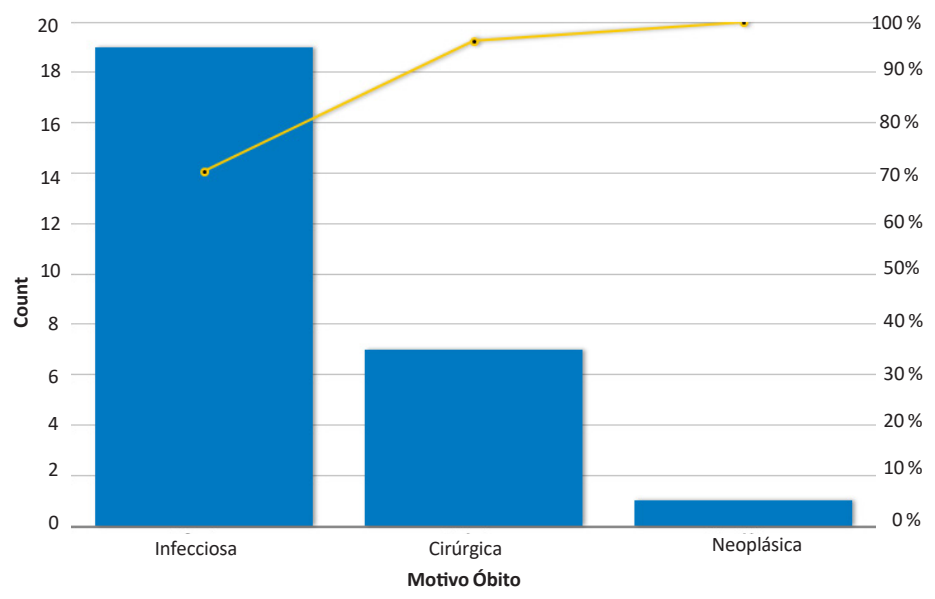

\section{DISCUSSÃO}

A política Estadual de Transplantes do Paraná, em desenvolvimento nos últimos oito anos, culminou com a marca de 50 doadores por milhão de população, no fechamento do primeiro semestre de 2018.11 Com o sedimentado crescimento no número de doadores, o próximo passo foi o início das discussões para estabelecimento de parâmetros e indicadores de resultados para monitoramento e avaliação dos serviços.

O desafio encontrado para a avaliação dos serviços foi a falta de dados que permitissem um diagnóstico correto da situação do estado, de maneira a respeitar as diferentes peculiaridades de cada centro transplantador.

O cenário atual de alocação de órgãos por critério de gravidade e os dados disponíveis no SNT permitem somente uma avaliação dos resultados pós-transplante utilizando o MELD como fator de estratificação de gravidade, índice que reconhecidamente possui diversas limitações para prever maior risco de morbimortalidade no período pós-transplante..$^{7,12}$

No âmbito da Câmara Técnica Estadual de Fígado, discutiram-se possíveis parâmetros a serem utilizados no acompanhamento dos resultados dos serviços transplantadores do estado, sendo que os parâmetros discutidos foram aplicados na coorte do Hospital do Rocio, com o intuito de apresentá-los por ocasião do I Congresso de Qualidade em Transplantes do Estado.

A análise dos dados coletados nos dois períodos não demonstrou diferenças em relação às características demográficas, porém observou-se significativo aumento do índice de gravidade representado pelo MELD (tabela 2), associado a um aumento da frequência de pacientes internados em ambiente de enfermaria e terapia intensiva no momento do transplante (figura 1).

A frequência de pacientes transplantados com pontos de exceção ao MELD manteve-se estável, em níveis baixos. Para fins comparativos, a média do MELD de pacientes transplantados, no início da implementação da nova política de transplantes do estado, em 2010, era de $18,36 \pm 7,25$ frente à atual: $24,4 \pm 7,11 .{ }^{13}$

A seleção de doadores manteve-se com características semelhantes nos dois períodos, sem diferenças significativas entre a idade, DRI, SOFA e o tempo de isquemia fria. (tabela 3 )

Índices de gravidade que envolvem fatores relacionados ao receptor e doador são os que melhor refletem a realidade da gravidade dos pacientes submetidos a transplante. Dentre esses, o escore de BAR, 8 PSOFT e SOFT6 são os mais validados na literatura.

A tabela 4 informa-nos a inexistência de diferenças significativas do BAR, PSOFT e SOFT entre os dois períodos. Houve diferença somente observada no escore DMELD, que é o produto da idade do doador e o MELD do receptor. A análise dessa diferença é simples, tendo sido impactada diretamente pelo significativo aumento do MELD entre os períodos, já que não observamos diferença entre as idades dos doadores.

A ausência de diferenças nos escores BAR, PSOFT e SOFT indica-nos que, no cômputo geral, a gravidade dos pacientes, em ambos os períodos, demonstrou-se semelhante, mesmo com a significativa diferença de MELD.

Por heterogêneas, a literatura traz como melhor validadas as complicações estratificadas segundo Clavien-Dindo. ${ }^{3} \mathrm{O}$ aumento da incidência no período de 2017 do Grau IVb e V (figura 2) reflete diretamente a maior morbimortalidade observada no período.

A incidência das complicações cirúrgicas específicas ao 
transplante de fígado manteve-se nos níveis da literatura (tabela 5).

Como ferramenta de controle de qualidade, o diagrama de Pareto demonstra que $20 \%$ das causas são responsáveis por $80 \%$ das consequências, isto é, há muitos problemas sem importância diante de outros mais graves. Aplicando esse princípio aos motivos de mortalidade cirúrgica, figura 3 , identificamos que complicação infecciosa foi responsável por $70 \%$ dos óbitos.

\section{CONCLUSÃO}

A adoção de acompanhamento prospectivo dos dados de transplante hepático no estado do Paraná, exemplificado agora pelos dados de um centro, permitirá uma análise mais fidedigna da realidade transplantadora em nosso estado. Projeções dos subgrupos de gravidade, segundo os índices adotados, servirão de base crítica para conhecimento de nossos limites, provendo diagnóstico mais preciso, transparência e melhor direcionamento de recursos do Sistema de Saúde.

\section{ABSTRACT}

Purpose: To identify and quantify complications in the first 30 days of cadaveric donor liver transplantation by using the Clavien-Dindo classification according to the DRI, P-SOFT, SOFT, MELD and BAR scores. To propose adoption of a prospective follow-up of these data in the Paraná State Transplant System. Methods: Observational cohort of THF cases from the Rocio Hospital program, Campo Largo, Paraná along 2016 and 2017. Results: a significant increase in the severity index represented by MELD with $20.2 \pm 4.8$ (2016) and 24. $4 \pm 7.11$ (2017), with $p=0.0076$. There were no significant differences in BAR $(8.03 \pm 3.86$ vs $9.54 \pm 4.11)$, PSOFT $(12.38 \pm 6.43$ vs $11.83 \pm 7.83)$ and SOFT $(12.32 \pm 7,6)$. There was an increased incidence of Grade IVb and V complications. The surgical mortality rate was $9.68 \%$ in 2016 , and $35.85 \%$ in 2017 . Conclusion: There was an increasing severity of MELD among receptors, with increased mortality due to infectious complications. The adoption of prospective follow-up of liver transplantation data in the state of Paraná, with projections of the severity sub-groups according to the adopted indices will serve as critical basis for continuous improvement, transparency and better targeting of health system resources.

Keywords: Liver Transplantation; Severity of Illness Index; Survival Rate; Benchmarking.

\section{AGRADECIMENTOS}

Hospital do Rocio e Central Estadual de Transplantes do Paraná.

\section{REFERENCES}

1. Busuttil RW. Transplantation of the liver. Third ed: Elsevier Saunders; 2015.

2. Diretrizes do Sistema Estadual de Transplantes do Paraná. Central Estadual de Transplantes do Paraná; 2012.

3. Dindo D, Demartines N, Clavien PA. Classification of surgical complications: a new proposal with evaluation in a cohort of 6336 patients and results of a survey. Ann Surg. 2004;240(2):205-13.
4. Clavien PA, Camargo CA, Jr., Croxford R, Langer B, Levy GA, Greig PD. Definition and classification of negative outcomes in solid organ transplantation. Application in liver transplantation. Ann Surg. 1994;220(2):109-20.

5. Feng S, Goodrich NP, Bragg-Gresham JL, Dykstra DM, Punch JD, DebRoy MA, et al. Characteristics associated with liver graft failure: the concept of a donor risk index. Am J Transplant. 2006;6(4):783-90. 
6. Rana A, Hardy MA, Halazun KJ, Woodland DC, Ratner LE, Samstein B, et al. Survival outcomes following liver transplantation (SOFT) score: a novel method to predict patient survival following liver transplantation. Am J Transplant. 2008;8(12):2537-46.

7. Brown RS, Jr., Kumar KS, Russo MW, Kinkhabwala M, Rudow DL, Harren P, et al. Model for end-stage liver disease and Child-Turcotte-Pugh score as predictors of pretransplantation disease severity, posttransplantation outcome, and resource utilization in United Network for Organ Sharing status 2A patients. Liver Transpl. 2002;8(3):278-84.

8. Dutkowski P, Oberkofler CE, Slankamenac K, Puhan MA, Schadde E, Mullhaupt B, et al. Are there better guidelines for allocation in liver transplantation? A novel score targeting justice and utility in the model for end-stage liver disease era. Ann Surg. 2011;254(5):745-53; discussion 53.

9. Freise CE, Gillespie BW, Koffron AJ, Lok AS, Pruett TL, Emond JC, et al. Recipient morbidity after living and deceased donor liver transplantation: findings from the A2ALL Retrospective Cohort Study. Am J Transplant. 2008;8(12):2569-79.
10. Dean AG AT, Sunki GG, Friedman R, Lantinga M, Sangam S, Zubieta JC, Sullivan KM, Brendel KA, Gao Z, Fontaine $\mathrm{N}$, Shu M, Fuller G, Smith DC, Nitschke DA, and Fagan RF. . Epi Info ${ }^{\mathrm{TM}}$, a database and statistics program for public health professionals. CDC, Atlanta, GA, USA. 2011.

11. АВTO. Registro Brasileiro de Transplantes. Associação Brasileira de Transplante de Órgãos; 2018. Contract No.: 2.

12. Desai NM, Mange KC, Crawford MD, Abt PL, Frank AM, Markmann JW, et al. Predicting outcome after liver transplantation: utility of the model for end-stage liver disease and a newly derived discrimination function. Transplantation. 2004;77(1):99-106.

13. Silveira FPS, F.; Macri, M. ; Nicoluzzi, JEL. Análise da mortalidade na lista de espera de fígado no Paraná, Brasil. O que devemos fazer para enfrentar a escassez de órgãos? ABCD, arq bras cir dig. 2012;25(2). 